\title{
ROTEIRO PARA O DESENVOLVIMENTO DE ASSENTOS INCLUSIVOS: a problemática do idoso
}

\author{
Maria Luíza Viégas Rodrigues Silva \\ Programa de Pós Graduação da Universidade Estadual de Minas Gerais (UEMG), Belo \\ Horizonte, Minas Gerais \\ luiza_viegas@hotmail.com \\ José Evandro Guimarães \\ Programa de Pós Graduação da Universidade Federal do Maranhão (UFMA). São Luís, \\ Maranhão \\ evaguim@hotmail.com
}

Resumo: Esta pesquisa possui como base a problemática do idoso diante da ação de sentar-se e levantar-se dos assentos. A discriminação do idoso manifesta-se também na inadequação dos mobiliários em relação às reais necessidades e fragilidades desse grupo e, pela existência de zonas estereotipadas, que são, por vezes, origens de estigmas, partindo do princípio que todo senil tem as mesmas necessidades, desejos e motivações que uma pessoa jovem. Foi definido como objetivo central deste trabalho a criação de recomendações para que possa facilitar a criação de cadeiras funcionais que possam ajudar os idosos a interagir com o seu meio sem riscos, auxiliando-os, ao mesmo tempo, facilitando o seu dia-a-dia. Pretende-se assim, conhecer o conceito real do idoso, das suas diferentes acepções e de que modo elas refletem na interação do idoso com o assento, e promover a importância desse objeto na vida dos idosos. A proposta então foi contemplada com uma apresentação que contém recomendações específicas para a problemática do assento para senis, com aspectos que são imprescindíveis para o processo de desenvolvimento de um produto com exigências tão sofisticadas.

Palavras-chave: Idoso, Assento, Cadeira, Metodologia, Design.

Abstract: This research has as its basis the problems of the elderly before
the action of sitting down and getting up the seats. The discrimination of
the elderly is also expressed in the inadequacy of securities in relation to the
real needs and weaknesses of this group, and the existence of stereotypical
zones, which are sometimes sources stigmas, assuming that everyone has
the same needs senile, desires and motivations that a young person. Was
defined as a central objective of this work to create recommendations that
would facilitate the creation of functional chairs that can help the elderly to
interact with their environment without risk, helping them at the same
time, facilitating the day-to-day. The aim is thus to know the real concept of 
the elderly, its various meanings and how they reflect the interaction of the elderly with the seat, and promote the importance of this object in life for seniors. The proposal was then covered with a presentation that contains specific advice on the issue of seat senile, with aspects that are essential to the development process of a product as sophisticated requirements.

Keywords: Elderly, Seat, Chair, Methodology, Design.

\section{INTRODUÇÃO}

De acordo com o censo realizado pelo IBGE (2010), 7,4\% da população brasileira eram pessoas acima de sessenta e cinco anos, e este índice cresce a cada ano a um nível sem precedentes. Segundo relatório da ONU (2007), uma previsão para 2050 é que um quarto da população mundial será de idosos, ou seja, teremos cerca de 2 bilhões de pessoas com idade acima de sessenta anos.

No Brasil ainda está sendo criado um mercado direcionado para idosos, porém no Japão, segundo HERWIG (2008), eles já reconheceram este mercado há muito anos, também chamado de "senior market", que cresce juntamente com o país. Os negócios e as indústrias japonesas trabalham com essa economia que possui muita força e faz circular a moeda. Das empresas como a Panasonic até as maiores empresas de cosméticos, os senhores e/ou idosos são respeitados e são considerados valiosos consumidores sendo atraídos por inovações e produtos anunciados por campanhas publicitárias.

Podemos perguntar-nos se o "crescimento" do número dos futuros aposentados é um fardo ou uma oportunidade para os designers e para a indústria. Coleman (2004) afirma que o efeito da melhoria das condições de vida, da nutrição e da medicina deve prolongar não só a expectativa de vida, mas também, facultar uma vida mais ativa para os aposentados. É natural pensar que os idosos possam permanecer economicamente independentes, gastando o seu dinheiro numa qualidade de vida melhorada e deste modo criar uma procura para novos bens e serviços. Seguindo este raciocínio, os "problemas" das pessoas idosas podem tornar-se numa oportunidade de negócio e estudo.

\section{DESENVOLVIMENTO}

\subsection{Design Inclusivo}

O design inclusivo muitas vezes é confundido com um design criado para situações específicas de pessoas com deficiência ou doentes, idosos ou crianças pequenas, mas não é isso. Tem sim por objetivo desenvolver produtos ou ambientes que possam ser utilizados por todas as pessoas sem que haja necessidade de qualquer adaptação específica, criando-se assim, e sendo criado, numa prática profissional com responsabilidade social.

Uma parte substancial dos projetos de design são desenvolvidos para pessoas de estatura e medidas antropométricas consideradas as ideais, sem condicionantes sensoriais ou de mobilidade. Esta é uma visão que não reflete a sociedade atual, que está envelhecendo, que inclui pessoas com deficiências ou com restrições temporárias.

Continuando com esta linha de pensamento, Santos (2007) refere que esta vertente do design ainda não faz parte do normal desenvolvimento do design, há poucos exemplos práticos de aplicação deste conceito. Mas todos podem usufruir dos benefícios do design inclusivo, que é substancialmente valorizado quando é 
desenvolvido com o propósito de integrar, quando se proporcionam as escolhas corretas, quando se projeta para e com as pessoas.

Implícito neste tipo de design está o fator ambiental, ao ser concebido um único design para todos, sem a necessidade de ter vários produtos para diferentes usuários, pode até ser considerado um design ecológico ou sustentável.

\subsection{Design Para Cadeiras}

Uma cadeira pode ter várias funções, mas a principal dela é que deve sustentar o corpo sentado sem se romper ou desarticular. A função principal é ter resistência. A armação ou estrutura de suporte deve ser suficientemente forte, o mesmo deve ocorrer com o assento e encosto, que devem suportar o peso quando o corpo se apoia sobre eles.

O tipo de sustentação de uma cadeira é determinado pelo seu uso. A cadeira pode ter variáveis formas, pode ser reta, incorporar braços, encosto para a cabeça ou para os pés, envolver o seu ocupante com almofadas, reclinar-se ou adaptar-se a diferentes posturas e alturas. Uma cadeira reta, que pode estar em torno de uma mesa, permite adotar uma postura mais cômoda para comer. Uma cadeira de escritório, em que se fica sentado por muitas horas diárias, deve permitir o movimento do corpo e evitar dores na coluna que aparecem quando se passa demasiado tempo na mesma postura. Já as cadeiras mais cômodas, pensadas para o relaxamento, podem ser reclináveis, de maneira que o peso modifica do assento ao encosto.

A profundidade e a altura são as medidas mais importantes no design de cadeiras, principalmente se forem utilizadas por pessoas com idades mais avançadas. Um assento demasiado alto deixa os pés flutuando e dar lugar a uma pressão excessiva nas curvas do corpo. Já um assento demasiado baixo, causa uma maior pressão sobre os ossos que sustentam a coluna vertebral. Um assento demasiado profundo nos empurra para trás, fazendo-se incomodo. As dimensões antropométricas aplicadas ao design de cadeiras permitem garantir que o peso se distribua de maneira confortável e segura para a saúde (DESIGN MUSEU, 2008).

\subsection{Antropometria do Assento}

O desenho do assento se remonta desde a Antiguidade. Segundo PANERO (2003), o assento apesar da sua ubiquidade e vida longa, continua sendo um dos piores elementos desenhados para o espaço interior. Uma das maiores dificuldades com que se tropeça nesta tarefa é que se entende que sentar-se é uma atividade estática, quando realmente é dinâmica. Assim, a aplicação exclusiva de dados bidimensionais estáticos na resolução de um problema tridimensional, que envolve aspectos biomecânicos, é uma abordagem errada. Paradoxalmente, uma cadeira antropometricamente correta, pode não ser confortável. No entanto, se o projeto simplesmente não atender a todas as dimensões humanas e corporais, não há duvida que o resultado cause desconforto no usuário.

O assento é provavelmente, uma das invenções que mais contribuiu para modificar o comportamento humano. Na vida moderna, muitas pessoas chegam a passar mais de 20 horas por dia nas posições sentadas e deitadas. lida (2003) cita que há diversas vantagens no ato de sentar, como: Consome menos energia, em relação à postura em pé e reduz a fadiga; Reduz a pressão mecânica sobre os membros inferiores; Reduz a pressão hidrostática da circulação nas extremidades e alivia o trabalho do coração; Facilita manter um ponto de referência para o trabalho (na posição em pé o corpo fica oscilando); Permite o uso simultâneo dos pés e das mãos. 
Porém, as desvantagens são: o aumento de pressão sobre as nádegas e a restrição dos alcances. Um assento mal projetado pode ocasionar estrangulamento da circulação sanguínea nas coxas e pernas.

lida (2003) afirma que para o assento ser confortável, é ideal colocarmos uma camada leve de estofamento, reduzindo a pressão máxima a cerca de $400 \%$ e aumentando a área de contato de 900 para $1050 \mathrm{~cm}^{2}$, sem prejudicar a postura mostra-se benéfica. $O$ estofamento então deve ser montado em uma base rígida para suportar o peso do corpo. Portanto, um estofamento pouco espesso, de 2 a $3 \mathrm{~cm}$, colocando sobre uma base rígida, que não se afunde com o peso do corpo, ajuda a distribuir a pressão e proporciona maior estabilidade ao corpo.

O material usado para revestir o assento deve ter característica anti-derrapante e ter capacidade de dissipar o calor e o suor gerado pelo corpo, não sendo recomendáveis plásticos lisos e impermeáveis.

Deve-se considerar também que muitos projetos são baseados nas respectivas normas técnicas. Cada país possui suas normas, além de que elas sejam frequentemente alteradas. No Brasil existem normas como a NBR 13962 e a NBR 14110.

Segundo lida (2003), existem seis princípios gerais sobre os assentos, derivados de diversos estudos anatômicos, fisiológicos e clínicos da postura sentada. Eles estabelecem também os principais pontos a serem considerados no projeto e seleção de assentos, como: As dimensões do assento devem ser adequadas às dimensões antropométricas do usuário; $O$ assento deve permitir variações de posturas; $O$ assento deve ter resistência, estabilidade e durabilidade; Existe um assento mais adequado para cada tipo de função; $O$ encosto e o apóia-braço devem ajudar no relaxamento; Assento e mesa formam um conjunto integrado.

Do ponto de vista antropométrico, a altura poplítea ou do sulco poplíteo (distância vertical entre o chão e a parte inferior da coxa, logo atrás do joelho) deveria ser medida como referência para estabelecer a altura adequada dos assentos. Segundo PANERO (2003), o valor mais baixo da tabela, como o percentil 5, deveria ser adequado desde que atenda o segmento da população com as menores dimensões corporais. PANERO (2003) sugere que a altura do sulco poplíteo seja para homens de $39,4 \mathrm{~cm}$ e $35,6 \mathrm{~cm}$ para as mulheres, sendo que essas medidas foram registradas sem nada nos bolsos, sem sapatos e utilizando uma túnica de exames. Indicando portando, um acréscimo de ambas as medidas de $3,8 \mathrm{~cm}$, para considerar diversos fatores, obtendo como resultados $43,2 \mathrm{~cm}$ para homens e $39,4 \mathrm{~cm}$ para mulheres. Já para IIDA (2003), os valores são entre no mínimo $35,1 \mathrm{~cm}$ ( $5 \%$ das mulheres), até no máximo 48 $\mathrm{cm}$ (95\% dos homens), pelas medidas tabeladas. Contudo, pode-se acrescentar mais $3 \mathrm{~cm}$ para a altura dos calçados $(38,1 \mathrm{a} 51 \mathrm{~cm})$.

$\mathrm{Na}$ antropometria, o comprimento nádega-sulco poplíteo (distancia horizontal da parte posterior da nádega até a parte posterior da perna, ou sulco atrás dos joelhos) é a medida ideal para estabelecer a profundidade adequada do assento. Conforme dita PANERO, um percentil 5 indica o comprimento nádega-sulco poplíteo de $43,9 \mathrm{~cm}$ para homens, e 43,2 $\mathrm{cm}$ para mulheres. Ou seja, uma profundidade de assento maior que 40,6 cm, não ira acomodar um usuário muito pequeno, enquanto para uma poltrona, uma profundidade de $43,2 \mathrm{~cm}$ acomodaria cerca de $95 \%$ da 
população de todos os usuários. A norma NBR 13962 recomenda a largura de $40 \mathrm{~cm}$ e profundidade útil de $38 \mathrm{~cm}$.

Os apóia-braços ou apoios de cotovelos também são usados continuamente, mas para os relaxamentos ocasionais. Servem para descansar os antebraços e ajudam a guiar o corpo durante o ato de sentar-se e levantar-se. Essa ajuda é importante principalmente para pessoas idosas e aquelas que possuem dificuldades de movimentar-se. A medida é dimensão do cotovelo até a superfície do assento. Panero (2003) cita que as maiores alturas atingem o percentil de 95 para homens com $29,5 \mathrm{~cm}$. Esta altura seria de fato desconfortável para a maioria das pessoas. 0 ideal seria 0 percentil 70, já que o 5 estaria abaixo do limite adequado. A maior parte das fontes, contudo, recomenda uma altura de apoio de braços entre 17,8 e 25,4cm.

\subsection{Idoso como Usuário}

Para entender o processo de envelhecimento é preciso analisar três aspectos principais onde estas modificações ocorrem. São eles os aspectos: socioeconômico, psicocognitivo e biológico/funcional. É importante salientar, ainda, que as diversas alterações, ocorridas no organismo dos idosos, podem variar de pessoa para pessoa, conforme suas condições físicas internas, o meio ambiente o qual está inserida e seu estilo de vida (SIMÕES, 1994, p.44). Quanto ao aspecto socioeconômico, as mudanças mais significativas estão relacionadas à aposentadoria, pois com o desligamento do trabalho há um afastamento de seu círculo social e, muitas vezes, uma redução no poder aquisitivo (MAZO et al., 2004). Quanto ao aspecto psicocognitivo, ocorrem alterações na inteligência, na memória, aprendizagem e no tempo de reação. Além disso, verifica-se um declínio nas aptidões psicomotoras relacionadas à coordenação, à agilidade mental e aos sentidos, afetando, por exemplo, seu desempenho em testes que exijam execução rápida de ações (BERGER, MAILLOUX-POIRIER, 1995).

Quanto ao aspecto biológico/funcional são evidenciadas alterações em sua aparência e em sistemas corporais complexos, como o sistema cardiovascular, pulmonar, músculo-esquelético e etc., ou ainda no sistema nervoso. Como por exemplo, as alterações no sistema cardiovascular tornam o idoso mais suscetível à fadiga, ao risco de queda, e falta de circulação em seus membros, entre outras consequências patológicas. Esse processo se inicia por volta dos 30 a 40 anos, mas acelera-se a partir dos 50 anos. As modificações no sistema músculo-esqueléticas são bastante comprometedoras, pois, com a diminuição da mobilidade e o enfraquecimento dos ossos, os idosos ficam mais suscetíveis a riscos de fraturas, quedas e acidentes (BRASIL, 2001). Nos sistemas sensoriais, evidencia-se uma diminuição na acuidade visual e auditiva, uma menor capacidade de adaptação a mudanças de temperatura ambiente, entre outras.

Segundo lida (2003), há uma redução maior na antropometria dinâmica durante o envelhecimento, observa-se uma gradativa perda de forças e de mobilidade, tornando os movimentos musculares mais fracos, lentos e de amplitude menor. Isso se deve aos processos de perda de elasticidade das cartilagens e da calcificação. Contudo, o sistema nervoso se degenera em uma velocidade menor, podendo haver um mecanismo de compensação à perda no sistema muscular.

Para agravar a vulnerabilidade da pessoa idosa no seu meio habitacional, observa-se que tanto o desenho como a construção da habitação, na sua maioria, não 
leva em consideração as necessidades dos moradores idosos em nível de segurança, localização, acessibilidade e independência (Holm 1998 apud Tanner et al., 2008). Além do mais Papaneck (1995 apud Daré, 2010), refere que a população idosa é exatamente o público-alvo mais esquecido pela indústria da construção e aos respectivos designers.

\subsection{Idoso e o Assento}

O envelhecimento traz, como uma de suas consequências, a diminuição do desempenho motor na realização das Atividades da Vida Diária (AVD), o que, entretanto, não leva as pessoas a se tornarem, necessariamente, dependentes de outros. Embora aproximadamente $25 \%$ dos idosos cheguem ao estado de dependência para realizar tarefas cotidianas (Spirduso, 1995), o que é uma parcela considerável da população para trazer problemas para a sociedade, essa situação não se traduz como a totalidade dos idosos, ou como uma condição que todos terão quando envelhecerem. Não obstante tal fato, as avaliações físicas da capacidade funcional de idosos, até o momento, são predominantemente dirigidas as pessoas com baixa condição física e com dificuldades para realizar AVD.

Sentar e levantar de uma cadeira ou de uma cama são apontados como requerimentos básicos para a manutenção de um estilo de vida independente nas pessoas idosas (Ikeda, Schenkman, Riley \& Hodge, 1991). Essas duas habilidades são classificadas como tarefas de transferência já que envolvem mudanças na posição corporal (Shumway-Cook \& Woollacott, 2007). Na literatura sobre tarefas de transferência existe um grande volume de trabalhos relacionados à ação de levantar (Bussmann \& Stam, 2002), porém poucos trabalhos interessados com a ação de sentar (Moraes, 1997). A dificuldade na realização dos movimentos de sentar e levantar são um fator de risco que pode predizer a probabilidade de ocorrência de quedas em idosos (Tinetti, Speechley \& Ginter, 1988). O melhor entendimento dos fatores afetando as ações de sentar e levantar em idosos tem implicações clínicas importantes, já que pode ajudar profissionais da saúde a avaliar e organizar programas de prevenção às quedas e de reabilitação.

As cadeiras disponíveis no mercado possuem assentos com diferentes modelos que incluem variações em altura, tamanho, inclinação, textura da superfície, firmeza, entre outros. A combinação destes atributos físicos é fator crítico no julgamento de quão fácil ou difícil é sentar e levantar da cadeira. De fato, no estudo conduzido por Munton, Ellis, Chamberlain e Wright (1981) sobre adequação de cadeiras, considera que os idosos têm como fatores importantes, em primeiro lugar, a facilidade para levantar, seguido pelo conforto, altura do assento e facilidade para sentar. A facilidade/dificuldade para sentar e levantar de uma cadeira está, provavelmente, muito dependente da variação da altura do assento. Sabe-se que a diminuição da altura do assento aumenta a necessidade de produção de força nos membros inferiores, particularmente na articulação do joelho (Rodosky \& cols., 1989). Esse aumento da demanda de força pode resultar na interpretação subjetiva de que a tarefa é mais difícil de realizar.

Há muita ênfase em parâmetros biomecânicos, e ignoram os aspectos perceptivos envolvidos com esses atos motores. Nenhuma ação é puramente motora, mas sim, envolve a combinação dos aspectos perceptivos e motores. Dessa forma, é imperativo entender como se dá a relação entre percepção e ação durante os atos de sentar e levantar. Considerando que a facilidade para sentar e levantar aparece no 
topo das reivindicações dos idosos em relação ao modelo da cadeira, torna-se necessário desenvolver estudos que avaliem aspectos perceptivos objetivamente.

Estudos envolvendo a percepção de affordances sugerem que a definição de qual altura do assento permite sentar ou não, está diretamente relacionada com a inspeção visual da cadeira e com as dimensões corporais, particularmente com o comprimento dos membros inferiores (Mark, 1987; Mark \& Vogele, 1987). Além disso, durante os movimentos de sentar e levantar a visão contribui para a percepção da posição dos segmentos corporais no espaço e das posições relativas entre eles.

Trabalhos com idosos sobre a percepção da posição articular indicam uma perda na capacidade de identificar o início dos movimentos articulares durante movimento passivo dos membros, bem como uma maior dificuldade em reproduzir o movimento passivo do membro (Kaplan, Nixon, Reitz, Rindfleish \& Tucker, 1985; Skinner, Barrack \& Cook, 1984). As pessoas idosas também exibem dificuldade em detectar a flexão e extensão máxima das articulações metacarpofalangeanas (Kokmen, Bossemeyer \& Williams, 1978).

\subsection{Métodos publicados}

A partir da análise de referências, para dar continuidade ao roteiro, foram pesquisadas metodologias já utilizadas como abordagem para desenvolvimento de produtos inclusivos para pessoas com capacidades reduzidas e até deficientes. As principais encontradas foram:

\subsubsection{USERfit}

A metodologia aplicada nesse estudo de caso já é utilizada por desenvolvedores para abordar a usabilidade em design, chamada USERfit (COLLEMAN, 2003). Que trabalha com um conjunto de nove ferramentas que combinam para ajudar no processo de recolha de desenho e informações obtidas usando uma variedade de técnicas de recolha de dados. A essência da metodologia é que ela proporciona uma estrutura para auxiliar o desenvolvedor garantir que as questões relevantes do projeto foram consideradas.

O desenho envolve geralmente certo número de elementos comuns. Normalmente, estes incluem: uma fase de definição do problema; o desenvolvimento de uma especificação funcional; uma fase de construção; uma fase de teste ou avaliação. Muitos designers reconheceriam intuitivamente esta sequencia, ainda que não utilizem realmente essas terminologias.

A fase da definição do problema é onde se identifica um problema e começar a conceituar uma solução em potencial - ou seja, a cadeira como produto destinado a resolver o problema. Este processo pode ser dividido em uma série de atividades interrelacionadas que diferem em propósito e nível de detalhes necessário. Estes podem incluir a investigação de base para estabelecer à natureza do mercado pretendido, produtos similares já existentes, a necessidade de aderir aos padrões, às necessidades dos usuários e seus constrangimentos perante o produto.

É nesta fase inicial do processo que se pode encontrar maior uso de ferramentas da metodologia de síntese. Ao todo, cinco das ferramentas podem ser utilizadas nesta fase. Resumidamente, no Contexto Ambiental e de Produtos há as Ferramentas do Ambiente que permitem refinar em uma compreensão geral do problema, que é o de ser explorado e a de examinar a forma como o produto pode se 
encaixar no ambiente mais amplo no qual os usuários finais vivem. O principal objetivo dessa ferramenta é forçar os futuros desenvolvedores a considerar as maiores implicações de como o produto será apoiado, e também algumas das prováveis implicações desses fatores.

Atividades de análise mais detalhadas são suportados por resumos de cobrir o que se sabe sobre as características dos usuários idosos (Análise de Usuário) e as suas atividades (Analises de Atividades) que eles precisam para executar. A Análise do Usuário atua como um repositório de informações de projeto sobre o usuário com características, e resume as implicações que estas podem ter para design. A Análise de Atividade desempenha uma função semelhante descrevendo as atividades ou tarefas que cada usuário precisa executar, sendo realizadas entrevistas, questionários e observações.

Descrevendo a especificação funcional inicial de um produto é suportada por Análise de Produto, que resume as decisões de design feitas quanto às características de um produto. Isto pode ser utilizado em uma variedade de formas, como diferentes tipos de atividade ditadas pelo design.

Uma vez que alguma compreensão dos usuários e de suas atividades tem sido obtida, é necessário que os desenvolvedores passem para o processo mais criativo de desenvolvimento de uma especificação detalhada de um produto para satisfazer necessidades de usuários. USERfit auxilia no processo de refinação de uma especificação funcional de produtos ou seja, o que deve ser implementado, mas não abordar explicitamente detalhes técnicos da especificação como um banco de dados pode ser organizada ou módulos de software construído.

O quadro USERfit auxilia neste processo de definição com um Produto Atributo Matrix, que ajuda os desenvolvedores em cruzar a características desejadas de um produto (como revelado por meio de Usuário e Análises de Atividades) com suas características reais (como sugerido através de Análise de Produto e as considerações Ambientais de Contexto). Isto permite que uma primeira análise do provável sucesso de um produto no cumprimento dos requisitos de usuários finais. Os resultados desta comparação são então utilizados para criar um Resumo de Requisitos e Resumo de projeto, que pode ser tomadas através no processo de criação.

A Construção do Produto segue o processo de especificação. A metodologia não presta assistência na gestão deste processo como uma grande atividade técnica e fora do âmbito do método. Muitos outros métodos estruturados já existem para apoiar essas atividades.

\subsubsection{Kano model}

No Modelo de Kano é defendido para alguns como atributos do produto e/ou serviço, a satisfação do cliente é aumentada drasticamente com somente uma pequena melhoria no desempenho, quando para outros atributos, a sua satisfação aumenta pouquíssimo quando o desempenho é melhorado ao extremo. Normalmente o modelo é aplicado para a classificação de atributos da qualidade; a ideia fundamental do modelo é que os atributos do produto e/ou serviço podem ser classificados em categorias conforme eles criam satisfação ou insatisfação com um nível de desempenho (HUISKONEN e PIRTTILÄ, 1998).

Kano et al. (1984), e Berger et al. (1995), sugerem que os atributos podem ser identificados perguntando aos consumidores sobre sua reação ou satisfação, quando os atributos têm desempenho superior (questão positiva) e inferior (questão negativa). 
Dependendo das respostas para a questão "positiva" e "negativa", o atributo pode ser identificado como atrativo, obrigatório, unidimensional, neutro ou reverso. Este modelo teórico se baseia na relação do grau de desempenho (eixo horizontal) com o grau de satisfação (eixo vertical), sendo que cada atributo resultante das diferentes relações entre os graus é descrito na sequencia (exceção para o atributo questionável, que não é representável no modelo teórico).

a) Atributo atrativo: este atributo é chave para a satisfação do cliente, se tiver alto grau de desempenho trará plena satisfação, porém, não trará insatisfação ao cliente se não for atendido;

b) Atributo obrigatório: se este não estiver presente ou se o grau de desempenho for insuficiente, o cliente ficará insatisfeito, por outro lado, se estiver presente ou tiver grau de desempenho suficiente, não trará satisfação;

c) Atributo unidimensional: quanto a este atributo, a satisfação é proporcional ao grau de desempenho, quanto maior o grau de desempenho, maior será a satisfação do cliente e vice-versa;

d) Atributo neutro: refere-se aos aspectos que não são bons e nem ruins, consequentemente, eles não resultam em qualquer satisfação ou insatisfação do cliente;

e) Atributo reverso: refere para o alto grau de desempenho resultando na insatisfação (e vice versa, o baixo grau de desempenho resultando na satisfação) e para o fato de não ser semelhante para todos os clientes;

f) Atributo questionável: esta avaliação indica que a pergunta foi formulada incorretamente, ou que o cliente não entendeu a pergunta corretamente, ou que a resposta foi inconsistente.

Conforme Sauerwein et al. (1996), o consumidor tende a dar maior importância aos atributos obrigatórios, decrescendo esta importância aos unidimensionais, atrativos e neutros, respectivamente. Para Matzler e Hinterhuber (1998), os atributos atrativos não são expressos pelo cliente, pois estas são as características que ele não espera.

Para se detectar os tipos de atributos, as classificações de Kano são identificadas através de um questionário especialmente projetado que contenha um par de perguntas para cada característica do produto e/ou serviço. A primeira questão refere-se à reação do cliente se o produto e/ou serviço tem essa característica (forma funcional da pergunta); a segunda refere-se a sua reação se o produto e/ou serviço não tem essa característica (forma disfuncional da pergunta).

Através da combinação das respostas para a forma funcional e disfuncional da pergunta, pode-se classificar, segundo as respostas dos clientes, um atributo em: atrativo, unidimensional, obrigatório, neutro, reverso ou questionável. Por exemplo, se a resposta do cliente para a questão funcional for "Eu espero que seja desta maneira" e se sua resposta disfuncional for "Eu não gosto disto dessa maneira", então, de acordo com a avaliação de Kano, a classificação segundo o cliente é atributo obrigatório.

A classificação dos atributos deve ser extraída para cada cliente entrevistado, tabulada e compilada. Os atributos classificados em obrigatórios, unidimensionais e atrativos são prioridades para desenvolvimento de um produto ou na prestação de um serviço. Por exemplo, não é muito vantajoso melhorar as exigências obrigatórias, visto que é um nível satisfatório; melhorar as exigências unidimensionais ou atraentes é 
mais vantajoso, uma vez que estas exigências têm uma maior influência na percepção da qualidade, por conseguinte no grau de satisfação do cliente (MATZLER e HINTERHUBER, 1998).

Ressalta-se que o Modelo de Kano não busca medir o grau de satisfação atual dos clientes com determinado atributo, no entanto, se os consumidores entrevistados forem clientes atuais do produto pesquisado, suas respostas serão influenciadas pelo desempenho atual, e isto deverá ser levado em consideração. Por exemplo, se a limpeza dos banheiros em um restaurante for inadequada, ao serem perguntados, os clientes atuais (que conhecem o estado das instalações) tenderão a classificar limpeza como atributo unidimensional. Já os possíveis consumidores, que não tiveram contato com a situação atual, tenderão a classifica-la como atributo obrigatório. Se os dois públicos forem misturados, as respostas poderão estar dispersas por diversas classes.

\section{CONCLUSÃO}

O objetivo geral do presente estudo foi criar um roteiro adequado para desenvolvimento futuro de uma cadeira que possa incluir socialmente idosos, analisando o efeito do envelhecimento nos aspectos perceptivos e motores envolvidos com as ações de sentar e levantar de uma cadeira. Este estudo recolheu informações necessárias sobre: o design e sua história, princípios do design universal e inclusivo, chegando ao design para cadeiras e sua história, antropometria de assentos para idosos e como o idoso se comporta neste meio.

Com base nesses preceitos foram analisados métodos já aplicados e estudados a fim de seguir um processo mais específico para a atual situação, os problemas que os idosos possuem em uma atividade básica e diária, o ato de sentar.

Assim, foi criado um roteiro para o desenvolvimento de assentos inclusivos, que nada mais é do que uma declaração de intenções. É um conjunto de restrições mentais que proporcionam a equipe de projeto uma referencia a partir da qual começar, benchmarks ${ }^{1}$ por meio dos quais é possível mensurar o progresso em um conjunto de objetivos a serem atingidos. Com relação ao produto a ser desenvolvido, é preciso conter uma primeira descrição de suas características e metas para o inicio do desenvolvimento, lançamento e retirada do mesmo.

Para a obtenção desses Requisitos foi utilizado o checklist baseado na proposta de Pugh apud Amaral (2006):

Desempenho: A cadeira em concepção deve satisfazer as necessidades do público alvo, ou seja, adultos a idosos, através do ato de sentar e levantar. Devendo ser avaliada antropometricamente e ergonomicamente a fim de evitar acidentes ou quedas.

Meio Ambiente: O produto passará por diversos processos mecânicos, químicos e físicos, com a finalidade de se obter o melhor meio de produção para o desenvolvimento dos assentos. Porém todo seu processo deverá ser limpo e minimizar ao máximo qualquer dano à natureza, sendo ecologicamente correto e seguindo normas ambientais. Podendo ser reutilizado materiais para a produção, e também levados à reciclagem ao final do ciclo de consumo.

Vida em Serviço: Será um bem de consumo durável, com ciclo de 5 a 15 anos.

\footnotetext{
${ }^{1}$ Benchmark: É um processo continuo de produtos ou serviços e práticas em relação aos mais fortes concorrentes ou empresas reconhecidas como lideres em suas indústrias. (Fonte: AMARAL, 2006)
} 
Eficiência: A cadeira deverá atender o maior nível de satisfação e eficiência possível, minimizando manutenção, já que o público alvo possa vir a ter alguma restrição motora. $\mathrm{A}$ relação custo benefício terá um nível mediano, e o produto deve transmitir alta confiabilidade.

Transporte: Processo todo feito através de containers e caminhões de transporte.

Embalagem: A priori a embalagem será de material reciclável, sem excessos.

Tamanho e Peso: 0 tamanho e peso da cadeira seria de acordo com estudos relacionados a postura sentada e a adultos e idosos, com analises antropométricas e ergonômicas. Seguindo as normas brasileiras e internacionais.

Estética, Aparência e Acabamento: A cadeira seguiria as preferências feitas por consumidores, através de uma pesquisa de mercado e entrevistas e questionários com os usuários, através da metodologia Userfit e do Modelo de Kano. Devendo seguir como principio o conforto e a comodidade.

Materiais: Materiais rígidos, estofados, parafusos etc. Devem ser laváveis, antichamas, recicláveis e que não agridam o meio ambiente.

Normas: NBR 13962, a NBR 14110, NBR ISO 10006:2000, NBR ISO 9001:2008

Ergonomia: Assentos com dimensões com normas antropométricas, alturas reguláveis, apoiabraços para relaxamento e utilização no ato de levantar e sentar, o assento deve permitir várias posturas, ter resistência e durabilidade etc.

Testes: Deverá ser analisada a cadeira com protótipos, testes físicos e mecânicos.

Ajustes no meio do caminho são comuns e representam uma característica natural de um processo saudável, flexivo e dinâmico. Esses ajustes contínuos do plano inicial ajudaram a orientar a equipe de projeto na direção do equilíbrio correto da praticabilidade, viabilidade e desejabilidade.

\section{REFERÊNCIAS}

AMARAL, Daniel Capaldo et al. Gestão de desenvolvimento de produtos. São Paulo: Saraiva, 2006.

BERGER L; MAILLOUX-POIRIER, D. Pessoas idosas -uma abordagem global. Lisboa (Portugal): Lusodidacta.1995.

COLEMAN, R., About: Inclusive Design. Disponível na internet em:

<http://www.designcouncil.org.uk/About-Design/Design-Techniques/ Inclusivedesign/>. 2004. Acessado em 26 de abril de 2012.

DARÉ, A. Design Inclusivo: o impacto do ambiente doméstico no utilizador idoso. Lisboa: Universidade Lusíada Editora. 2010 HUISKONEN, J.; PIRTTILÃ, T. Sharpening logistics customer service strategy planning by applying Kano's quality element classification. Production Economics, v.56-57, p.253-260,1998.

IIDA, Itiro. Ergonomia: projeto e produção. 2aed. São Paulo: Blucher, 2005 IKEDA, E. R., Schenkman, M. L., Riley, P. O., \& Hodge, W. A. Influence of age on dynamics of rising from a chair. Physical Therapy, 71, 473-481. 1991. 
INSTITUTO BRASILEIRO DE GEOGRAFIA E ESTATÍ́STICA (IBGE). Estudos \& pesquisas informação demográfi ca n.9: perfi I dos idosos responsáveis pelos domicílios no Brasil 2010. Ministério do Planejamento, Orçamento e Gestão. Rio de Janeiro: IBGE, 2002. Disponível na internet em: <http://www.ibge.gov.br/> Acessado em: 10 abril 2012.

KANO, N. et al. Attractive quality and must-be quality, Hinshitsu, v.14, n.2, p.147-56, 1984.

KAPLAN, F. S., Nixon, J. E., Reitz, M., Rindfleish, L., \& Tucker, J. Age-related changes in proprioception and sensation of joint position. Acta Orthopaedica Scandinavica, 56, 72-74. 1985

KOKMEN, E., Bossemeyer, R. W. Jr., \& Williams, W. J. Quantitative evaluation of joint motion sensation in an aging population. Journal of Gerontology, 33, 62-67.1978. MARK, L. S. Eyeheight-scaled information about affordances: A study of sitting and stair climbing. Journal of Experimental Psychology: Human Perception and Performance, 13, 361-370. 1987.

MORAES, R. Efeitos do envelhecimento nas habilidades de andar para frente, andar para trás, sentar e levantar. Tese (Mestrado), Universidade Estadual Paulista, Rio Claro. 1997.

MATZLER, K.; HINTERHUBER, H. H. How to make product development projects more successful by integrating Kano's model of customer satisfaction into quality function deployment. Technovation, v.18, n.1, p.25-38, 1998.

MUSEUM, Design. Cómo diseñar una silla. Barcelona: Editora Gustavo Gil. 2008. MUNTON, J. S., Ellis, M. I., Chamberlain, M. A., \& Wright, V. An investigation into the problems of easy chairs used by the arthritic and the elderly. Rheumatology and Rehabilitation, 20, 164-173. 1981

PANERO, J.; ZELNIK, M. Dimensionamento humano para espaços interiores.

Barcelona: Gustavo Gili, 2003.

RODOSKY, M. W., Andriacchi, T. P., \& Andersson, G. B. The influence of chair height on lower limb mechanics during rising. Journal of Orthopaedic Research, 7, 266-271. 1989

SANTOS, R. \& SENNA, C. \& VIEIRA, S. Acessibilidade E Design Inclusivo - Um estudo sobre a aplicação do design universal nos produtos industriais. Seminário de Produção Académica em Design, Florianópolis.2007.

SAUERWEIN, E. et al. The Kano model: how to delight your customers. International Working Seminar on Production Economics, v.1 of the IX, Austria, p.313-327, Feb., 1996.

SHUMWAY-COOK, A., \& Woollacott, M. H. Motor control: Translating research into clinical practice. Philadelphia: Lippincott Williams \& Wilkins. 2007.

SIMÕES, Regina. Corporeidade e Terceira Idade: a marginalização do corpo idoso. Piracicaba: Unimep, 131p. 1994.

SKINNER, H. B., Barrack, R. L., \& Cook, S. D. (1984). Age-related decline in proprioception. Clinical Orthopaedics and Related Research, 184, 208-211. 1984. SPIRDUSO, W. W. Physical dimensions of aging. Champaign: Human Kinetics. 1995. TINETTI, M. E., Speechley, M., \& Ginter, S. F. Risk factors for falls among elderly persons living in the community. The New England Journal of Medicine, 319, 17011707.1988. 